\title{
Establishing a surgical skills laboratory and dissection curriculum for neurosurgical residency training
}

\author{
James K. C. Liu, MD, Varun R. Kshettry, MD, Pablo F. Recinos, MD, Kambiz Kamian, MD, \\ Richard P. Schlenk, MD, and Edward C. Benzel, MD
}

Department of Neurosurgery, Neurological Institute, Cleveland Clinic, Cleveland, Ohio

\begin{abstract}
Surgical education has been forced to evolve from the principles of its initial inception, in part due to external pressures brought about through changes in modern health care. Despite these pressures that can limit the surgical training experience, training programs are being held to higher standards of education to demonstrate and document trainee competency through core competencies and milestones. One of the methods used to augment the surgical training experience and to demonstrate trainee proficiency in technical skills is through a surgical skills laboratory. The authors have established a surgical skills laboratory by acquiring equipment and funding from nondepartmental resources, through institutional and private educational grants, along with product donations from industry. A separate educational curriculum for junior- and senior-level residents was devised and incorporated into the neurosurgical residency curriculum. The initial dissection curriculum focused on cranial approaches, with spine and peripheral nerve approaches added in subsequent years. The dissections were scheduled to maximize the use of cadaveric specimens, experimenting with techniques to best preserve the tissue for repeated uses. A survey of residents who participated in at least 1 year of the curriculum indicated that participation in the surgical skills laboratory translated into improved understanding of anatomical relationships and the development of technical skills that can be applied in the operating room. In addition to supplementing the technical training of surgical residents, a surgical skills laboratory with a dissection curriculum may be able to help provide uniformity of education across different neurosurgical training programs, as well as provide a tool to assess the progression of skills in surgical trainees.
\end{abstract}

http://thejns.org/doi/abs/10.3171/2014.11.JNS14902

KEY WORDS surgical skills laboratory; residency education; anatomical dissection

$\mathrm{T}$ HE establishment of formal surgical training in the US originated with William Stewart Halsted when he established the training program at The Johns Hopkins Hospital in 1889. According to Halsted's philosophy, surgical training was predicated on an apprenticeship model based on several principles, among which included a close association between the resident and the professor, as well as an "indefinite tenure" of training.? Recently, greater scrutiny of residency education and patient safety has led to necessary modifications in how physicians are trained..$^{5}$ Duty-hour limitations established by the Accreditation Council of Graduate Medical Education (ACGME) have caused all medical specialties to reevaluate their educational curriculum, but surgical specialties may have been the most affected. Duty-hour constraints have led many neurosurgical senior residents and program directors to acknowledge that these limitations have had an adverse effect on the surgical training experience. ${ }^{4,8}$ Pressures to improve patient safety, outcomes, and hospital efficiency are also likely to exert an effect on residency education by limiting surgical autonomy. ${ }^{3}$ The combination of these external pressures has caused training programs to reevaluate their approach to surgical education to improve the efficiency of surgical training. One strategy is to employ more hands-on training by incorporating environments into residency training that simulate the operating room experience.

To comply with the newly developed mandates on surgical education, neurosurgical training programs will likely need to develop strategies for systematically assessing a trainee's surgical competency prior to graduation. The best way to develop these skills and perform such an as-

ABBREVIATIONS ACGME $=$ Accreditation Council of Graduate Medical Education; PGY = postgraduate year.

SUBMITTED April 21, 2014. ACCEPTED November 6, 2014.

INCLUDE WHEN CITING Published online May 26, 2015; DOI: 10.3171/2014.11.JNS14902.

DISCLOSURE The Cleveland Clinic surgical skills laboratory is supported in part by an educational grant from Stryker. In addition, previous product donations and grants for the laboratory have been obtained from Covidien, Medtronic, Synthes Anspach, and Aesculap. 
sessment in a controlled and safe atmosphere may be in an anatomical dissection laboratory. Anatomical dissection laboratories have long been a part of the training process for neurosurgeons wishing to master microsurgical techniques. ${ }^{12,16}$ Although many neurosurgeons have used the dissection laboratory to develop their skills, structured dissection curricula have yet to become a staple in medical education. There are multiple barriers to instituting a surgical skills curriculum, including space, funding, and equipment. ${ }^{13}$

Our institution (Cleveland Clinic) recently conducted a survey of all (100) neurosurgical program directors in the US to assess the current role of anatomical dissection in residency training. ${ }^{11}$ Of the 65 training programs $(65 \%)$ that responded to our survey, 61 (93.8\%) had cadaveric or animal dissection available in their training program, but only $58.3 \%$ had scheduled dissection sessions with additional laboratory availability for independent study. Of the responders, $95.4 \%$ believed that an anatomical dissection curriculum should be an integral part of surgical training, and $89.2 \%$ would support the creation of a uniform national dissection curriculum for all programs. Thus, there does appear to be a role for anatomical dissection, but it has yet to become implemented across all training programs.

In this paper we have outlined our experience in constructing a fully equipped surgical skills laboratory for anatomical dissection, established predominantly from extradepartmental sources. We have also been able to design and incorporate a dissection curriculum into the residency educational curriculum, creating a structured learning system for surgical approaches that we believe should be mastered by all neurosurgical residency graduates. We also report a survey completed by Cleveland Clinic neurosurgical residents who have completed at least 1 year of the dissection curriculum to assess the impact of incorporating a dissection curriculum into the surgical education experience. This impact was also measured through a survey of neurosurgical attending physicians at our institution.

\section{Methods}

\section{Laboratory Environment}

Initial expansion of the surgical skills laboratory was performed with minimal departmental startup funds. We obtained most of our equipment by applying for in-kind donations from industry. We also applied for educational grants from industry as well as internal institutional programs to obtain funding to purchase equipment that was not available through donations.

Securing adequate space is possibly the most crucial, yet difficult, step in establishing a surgical skills laboratory. One of the keys to a successful surgical skills laboratory is the ability to accommodate multiple dissections, with no more than 2 surgeons per station. For new dissection laboratories, obtaining space in or near an existing wet laboratory that might also share these facilities increases efficiency. A proper wet laboratory space that is equipped to handle chemical compounds and biohazardous materials should possess proper ventilation, a sink for cleaning instruments, and freezers or cold rooms reserved for cadaveric specimen storage.
The dissection stations should mimic the operating room environment. This includes an operating microscope, vacuum aspirator, high-speed electric drill, Mayfield head clamp with a tabletop mount, and basic surgical and microsurgical instruments. We believed it imperative that one of the surgical stations could serve as a "master station," to be be used for demonstration purposes. The microscope at this station was attached to a beam splitter and camera, which projected the microscopic view onto a large monitor. Also attached was a video capture unit to allow for recording of both still images and video for archival purposes. Much of this equipment can be purchased as refurbished products from local distributors at a significantly discounted price.

\section{Operating Microscopes}

A functioning operating microscope with good optics and adequate lighting is the keystone of a surgical skills laboratory dissection station. There are several methods of obtaining a microscope on a budget. The easiest is to search for older models that have been retired within one's institution. Alternatively, microscope distributors often have a stock of older models that they receive when a hospital purchases a new microscope. If a hospital has a relationship with these distributors, they may be willing to refurbish and either discount or donate these microscopes to a laboratory. Finally, microscope manufacturers or distributors may be willing to donate a new tabletop microscope when an institution purchases a new microscope. In our laboratory, we were able to obtain 2 refurbished microscopes from our local distributor at no charge. We were also able to obtain 2 retired microscopes from our institution.

\section{High-Speed Drills}

High-speed electric drills with multiple attachments are necessary to simulate skull base drilling. Many companies are willing to donate refurbished drills. Drill bits may be obtained through donations. In addition, lightly used bits from the operating room may be a possibility. One method to reduce the variety of drill bits needed is to use drills that use a single-length drill bit that fits all attachments. In our laboratory, we use the Stryker CORE Universal drill system (Stryker Corporation). This system accommodates different-length attachments on the drill handle while using the same-length drill bit. This allows one to use the same type of bit for drilling at different lengths, from short to endonasal drilling. Automated irrigation attachments for drills are also invaluable for deep skull base drilling.

\section{Additional Components}

Other components necessary for the dissection station can be obtained through donations or purchase. Wall suction is ideal but can be substituted with portable electric aspirators. We use portable electric aspirators (Schuco Aspirator Model S330A) with reusable canisters, which provide adequate suctioning during skull base drilling. Mayfield head frames and tabletop clamps are needed to secure the head in the desired position for dissection, although foam padding can be used. Basic surgical and 
microsurgical dissection instruments can generally be acquired through donations. Another cost-saving alternative is to purchase instruments from veterinary surgical instrument companies, particularly those offering microinstruments for veterinary ophthalmological surgery.

\section{Cadaveric Specimens}

Freshly embalmed and injected cadaver specimens are the key to maximizing the dissection experience. Injected cadaveric specimens can be obtained from the state anatomy board or from private companies who prepare specimens for dissections. Certain companies offer latex injection of the vasculature. Most medical centers associated with a medical school have arrangements for specimen cremation of their cadavers. The ability to use these services reduces return shipping and cremation costs. The key to an excellent vasculature injection is early washing (within 24 hours) of the vascular tree postmortem. If purchasing specimens from a company, it is prudent to inquire about the usual postmortem interval before vascular washing and fixation. The other key to a good injection is assessment of the specimen's vasculature during washing. For example, severely stenotic vertebral arteries may benefit from a thinner injection solution unless there is brisk flow across the posterior communicating artery during carotid artery washing. Good injections can be obtained with either silicone or latex. ${ }^{2,14,15}$

To maximize specimen use, we have experimented with different methods of specimen storage for future use. Freezing the specimen between uses requires thawing of the specimen several days prior to the laboratory session, and there is clear degeneration of the tissue. In our experience, optimal storage was obtained using $66 \%$ ethyl alcohol solution in a bucket and placed into a cooler at $40^{\circ}-50^{\circ} \mathrm{F}$. Specimens lasted significantly longer when placed in a cooler and were sufficiently malleable when removed several hours prior to dissection. We also kept a number of specimens in ethyl alcohol at room temperature for resident ad hoc use. When higher concentrations of ethyl alcohol were used (90\%-100\%), we found that parenchymal volume loss was accelerated. Ethyl alcohol also has a tendency to stiffen parenchyma. In lightly fixated specimens, this was advantageous, but if using heavily fixed specimens, one may need to either reduce the concentration of ethyl alcohol or add fabric softener. ${ }^{10}$

\section{Dissection Curriculum}

To incorporate the skills laboratory into the residency educational curriculum, we reserved 4 hours of protected time each month for the laboratory. We divided the residents into junior- (postgraduate year [PGY] 2-3) and senior-level (PGY 4-5) resident groups and created a dissection curriculum tailored to each group. The junior curriculum was tailored to focus on anatomy and basic surgical techniques and approaches. The senior curriculum focused on advanced skull base approaches. The dissection curriculum was designed to provide a comprehensive, anatomically oriented approach to the cranial cavity from anterior to posterior. Each session begins with a 45-minute didactic session reviewing the pertinent anatomy and techniques, followed by the dissection portion.
An entire 9-session cranial curriculum can be designed around a minimum of 2 sets of cranial specimens (Table 1). If funding permits, designing the curriculum around 3-4 specimens allows for residents to practice on unused sides on their own time. When designing a curriculum, there are some important considerations. First, approaches involving dissection of parenchyma (e.g., an interhemispheric transcallosal approach) should be performed early after specimen arrival. Pure bone dissections (e.g., a posterior petrosal approach) should be performed last. Materials should be planned accordingly. For example, we placed a session on carotid endarterectomy and microvascular anastomosis after pterional and peripheral nerve sessions to allow radial artery harvest and to have the carotid and middle cerebral artery exposures already completed. Complexity should be alternated to allow for even spacing of PGY 2-3 and 4-5 sessions. Finally, if available at one's institution, collaboration with neurootologists and rhinologists for posterior petrosal and endoscopic endonasal sessions, respectively, can be of major educational value.

After the first year, we expanded the curriculum to include dissections in the spine and peripheral nerves. Peripheral nerves, in particular, are an area of neurosurgery to which many residents have insufficient exposure. Laboratory dissection is an avenue by which programs can help supplement deficiencies. We obtained 3 fresh-frozen torso specimens that were intact from the head to the pelvis with a single arm (approximately $\$ 2500 /$ specimen + shipping). We arranged 5 dissection sessions around this single set of specimens covering the occiput to ilium (Table 2). Sessions were pooled into weekly sessions over 5 weeks. Specimens were placed in a $4^{\circ} \mathrm{C}$ cooler in between sessions. Combining too much material in 1 or 2 sessions results in mental fatigue and poor recall. We found this arrangement to be a good balance between allowing time for preparation, learning, and specimen preservation.

\section{Resident and Faculty Survey}

The dissection curriculum at the Cleveland Clinic Neurosurgery Program has been instituted since the 20112012 academic year. We collected an anonymous survey from all the residents who have participated in the curriculum since its inception. We also surveyed attending neurosurgeons in the department that have been faculty since the inception of the dissection curriculum regarding their assessment of changes in surgical skill level of the residents in the operating room.

\section{Results}

The dissection curriculum was implemented in the 2011-2012 academic year. The curriculum consisted of 4 sessions for junior residents and 5 sessions for senior residents. In the 2nd year, we incorporated peripheral nerve and spinal instrumentation sessions (Fig. 1).

We collected results from all current residents in our program who had undergone the dissection curriculum since its inception (Fig. 2). Twelve residents responded to the survey. They ranged from PGY 2 to PGY 7 at the time of taking the survey. All the residents agreed that dividing the sessions by junior- and senior-level groups was appro- 
TABLE 1. Sample 9-session cranial curriculum using 2 sets of specimens*

\begin{tabular}{|c|}
\hline Specimen 1 \\
\hline Session I: Basics of craniotomy, pterional approach (PGY 2-3) \\
\hline Pinning and positioning \\
\hline Temporalis muscle anatomy and dissection \\
\hline Pterional craniotomy \\
\hline Session II: Orbitozygomatic craniotomy (PGY 4-5) \\
\hline 1- vs 2-piece orbitozygomatic craniotomy \\
\hline Pretemporal approach \\
\hline Extradural anterior clinoidectomy \\
\hline Transsylvian approach and circle of Willis anatomy \\
\hline Session III: Far-lateral and telovelar approach (PGY 2-3) \\
\hline Suboccipital craniectomy and C-1 laminectomy \\
\hline Telovelar approach to the 4th ventricle \\
\hline Far-lateral craniectomy \\
\hline Vertebral artery and cerebellomedullary anatomy \\
\hline Session IV: Carotid endarterectomy, microvascular anastomosis (PGY 4-5)† \\
\hline Anterior cervical neck carotid anatomy \\
\hline Carotid endarterectomy technique \\
\hline Microvascular anastomosisł \\
\hline Session V: Retrosigmoid approach (PGY 2-3) \\
\hline Venous sinus anatomy \\
\hline Retrosigmoid craniectomy \\
\hline Cerebellopontine anatomy \\
\hline Specimen 2 \\
\hline Session VI: Approach to the lateral and 3rd ventricle (PGY 2-3) \\
\hline Anterior interhemispheric transcallosal approach \\
\hline Posterior interhemispheric transcallosal approach \\
\hline Contralateral transfalcine transprecuneal approach to the atrium \\
\hline Lateral and 3rd ventricular anatomy \\
\hline Session VII: Middle fossa approach (PGY 4-5) \\
\hline Temporal craniotomy and dural dissection \\
\hline Middle fossa anatomy \\
\hline Anterior petrosectomy \\
\hline Session VIII: Endoscopic endonasal approach to the sella (PGY 2-3) \\
\hline Nasal anatomy \\
\hline Endoscopic endonasal approach \\
\hline Sellar and parasellar anatomy \\
\hline Expanded transplanum approach \\
\hline Session IX: Posterior petrosal approach (PGY 4-5) \\
\hline Temporal bone anatomy and drilling technique \\
\hline Retrolabyrinthine approach \\
\hline Translabyrinthine approach \\
\hline Transcochlear approach \\
\hline
\end{tabular}

\footnotetext{
* Two is the minimum number of specimens needed to complete the entire curriculum. If funding allows, additional specimens can be used and unused portions may be saved for additional resident practice.

$\dagger$ Injected fixated specimens still allow good practice in suturing technique, but if funding allows, consider using fresh noninjected specimens to better mimic tissue characteristics when performing anastomosis.

$\ddagger$ We use a radial artery harvested from the torso laboratory series and practice high-flow internal carotid artery to middle cerebral artery anastomoses using exposed arteries from prior pterional/orbitozygomatic laboratory dissections.
} 
TABLE 2. Sample curriculum for a single-torso cadaver with 1 upper extremity

\begin{tabular}{|c|}
\hline Session I: Peripheral nerve (PGY 2-5) ${ }^{\star}$ \\
\hline Brachial plexus \\
\hline Radial nerve exposure \\
\hline Cubital tunnel \\
\hline Carpal tunnel \\
\hline Guyon's canal \\
\hline Session II: Thoracic spine (PGY 4-5) \\
\hline Thoracic laminectomy \\
\hline T2-12 thoracic pedicle screw fixation \\
\hline Transpedicular approach \\
\hline Costotransversectomy \\
\hline Lateral extracavitary approach \\
\hline Session III: Cervical spine (PGY 2-3) \\
\hline Anterior cervical discectomy/corpectomy and plate fixation \\
\hline Keyhole foraminotomy \\
\hline C3-6 cervical laminoplasty/laminectomy \\
\hline C3-6 lateral mass fixation \\
\hline T-1 pedicle screw fixation \\
\hline Session IV: Lumbar spine (PGY 2-3)† \\
\hline Microdiscectomy \\
\hline Foraminotomy \\
\hline Laminectomy \\
\hline Pedicle screw fixation \\
\hline Open TLIF \\
\hline Session V: Cervical spine (PGY 4-5) $\ddagger$ \\
\hline Posterior C1-2 fixation \\
\hline Direct lateral approach \\
\hline Transoral approach \\
\hline $\begin{array}{l}\text { TLIF = transforaminal lumbar interbody fusion. } \\
\text { * These sessions are suitable for all PGYs } 2-5 \text {. } \\
\text { † A PGY } 4-5 \text { session could include minimally invasive TLIF, Ponte osteoto- } \\
\text { mies, pedicle subtraction osteotomy, and iliac screw fixation. } \\
\text { ‡ Alternatively, this session can be completed using cranial specimens to } \\
\text { allow easy transition from posterior to lateral to anterior positions. }\end{array}$ \\
\hline
\end{tabular}

priate for trainee skill level. The most valuable aspect of the sessions was mastering anatomical relationships and surgical approaches. This was followed by gaining more experience with surgical instruments and equipment, and gaining experience with less commonly performed procedures. Eleven residents (92\%) agreed that the laboratory sessions improved their ability to understand anatomical relationships, and 8 residents $(67 \%)$ believed that the dissection sessions improved their technical skills in the operating room. Eight residents (67\%) stated that they have been able to apply concepts and techniques they learned in the dissection sessions in the operating room, and 11 residents $(92 \%)$ believed that being able to perform a procedure in the dissection laboratory would enhance the learning experience prior to performing actual surgery. The residents were also surveyed regarding their thoughts on duty-hour restrictions (Fig. 3). Only 4 residents (33\%) believed that duty hours had hindered their ability to develop their surgical skills. Of those residents, all stated that the dissection laboratory effectively supplemented the operating experience.

Nine attending neurosurgeons in the Department of Neurosurgery responded to the faculty survey (Fig. 4). Five surgeons (55.6\%) indicated a significant improvement in operative skill level since the implementation of the dissection curriculum, 3 (33.3\%) noted a slight improvement, and $1(11.1 \%)$ stated there was no change. Areas of improvement that were noted were use of the high-speed drill, followed by understanding of 3D anatomy and general microsurgical techniques. Areas in which the surgeons would most like to see further improvement through utilization of the laboratory included understanding of 3D anatomy and surgical-approach planning. Five of the 9 respondents stated that they used a dissection laboratory during their residency, but all agreed that it should be part of the current training program and that residents should demonstrate proficiency with skills and/or procedures in the laboratory prior to performing them in the operating room (Fig. 4).

\section{Discussion}

Anatomical dissections have always provided an avenue for fully trained neurosurgeons to enhance their anatomical knowledge and microsurgical skills. ${ }^{1,9}$ Yaşargil noted that, as a young surgeon, he had a mental barrier to skull base surgery because he felt uncomfortable and inexperienced with skull base anatomy and high-speed drill technology, which he ameliorated through his experience in the dissection laboratory. ${ }^{16}$ It is only logical that this component of training should become a component of the surgical educational experience. For training programs without a surgical skills laboratory already in place, the establishment of one may seem onerous. We have outlined a strategy to do so using our experience with minimal starting resources.

The addition of a surgical skills laboratory can augment the surgical training experience. Trainees will be able to gain a better understanding of surgical anatomy and approaches without the constraints of the operating room atmosphere. For advanced-level trainees, a surgical skills laboratory can help to better prepare them prior to performing a complex procedure in the operating room, thus maximizing the learning potential for less commonly performed procedures. In our survey of residents who have participated in the dissection curriculum since its inception at our residency program, nearly all of the participants agreed that the dissection sessions could be beneficial in this regard. We believe the keys to achieving this are to divide the sessions by resident training level and to maintain a low resident-to-cadaver ratio, which allows for adequate hands-on dissection time.

Nearly all of the attending surgeons noted improvements in surgical skill level since the introduction of the dissection curriculum, although the subjectivity of this assessment method makes it difficult to adequately judge the impact of the curriculum. Standardized motor-skill assessment tools may be implemented in the future to fully assess whether a dissection curriculum truly has an impact. Despite the fact that nearly half of the attending surgeons 

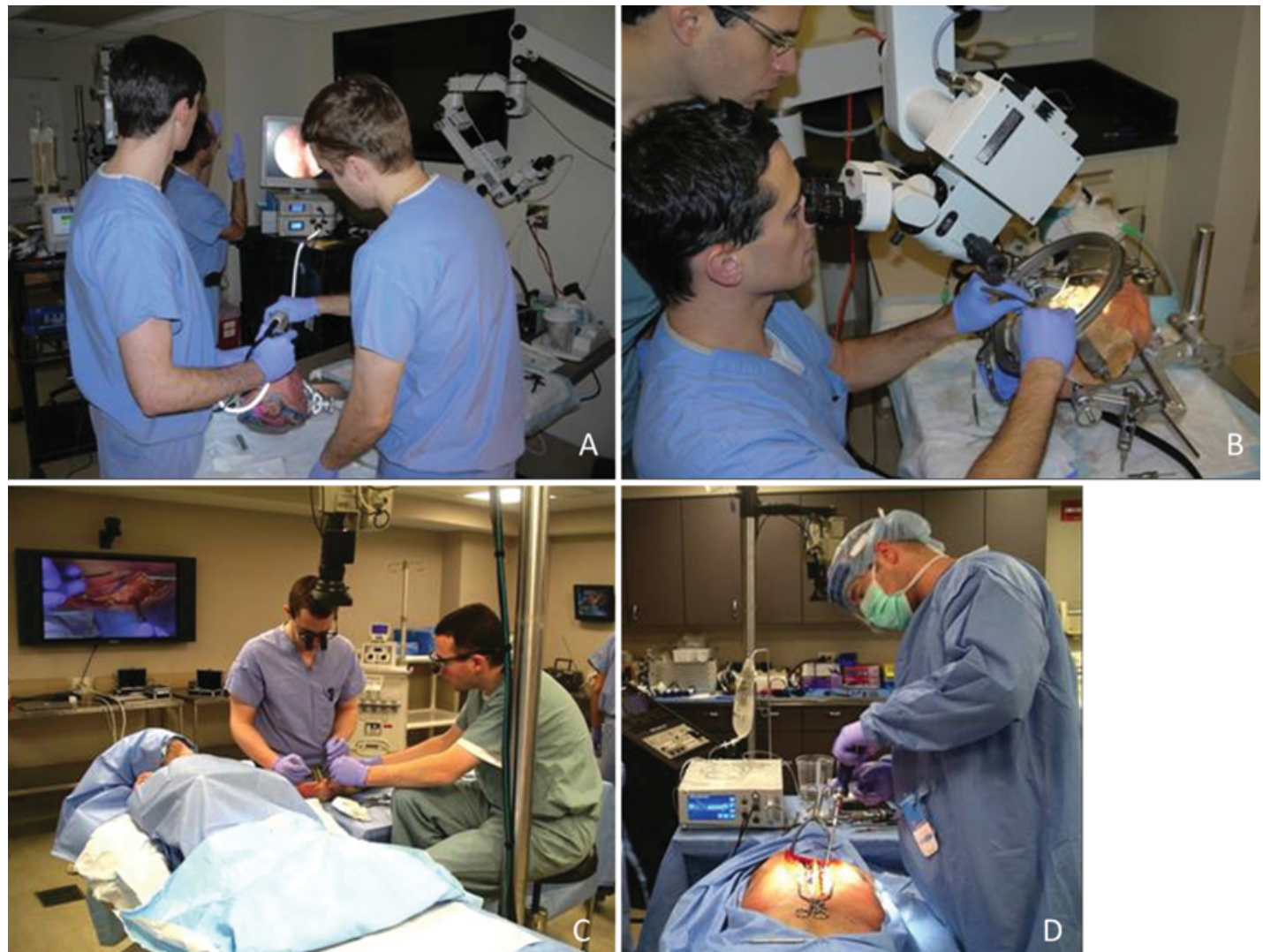

FIG. 1. Photograph of endoscopy (A), general skull base (B), peripheral nerve (C), and spinal instrumentation (D) dissection sessions in the surgical skills laboratory. Figure is available in color online only.

in our survey did not have access to a dissection laboratory during their residency, all believed that it should be part of the current training program. Implementing such programs can lead the way to greater uniformity among training programs despite variations in surgical volume and expertise available at individual programs. Although nothing will replace the experience and knowledge gained from the actual operative experience, this method will

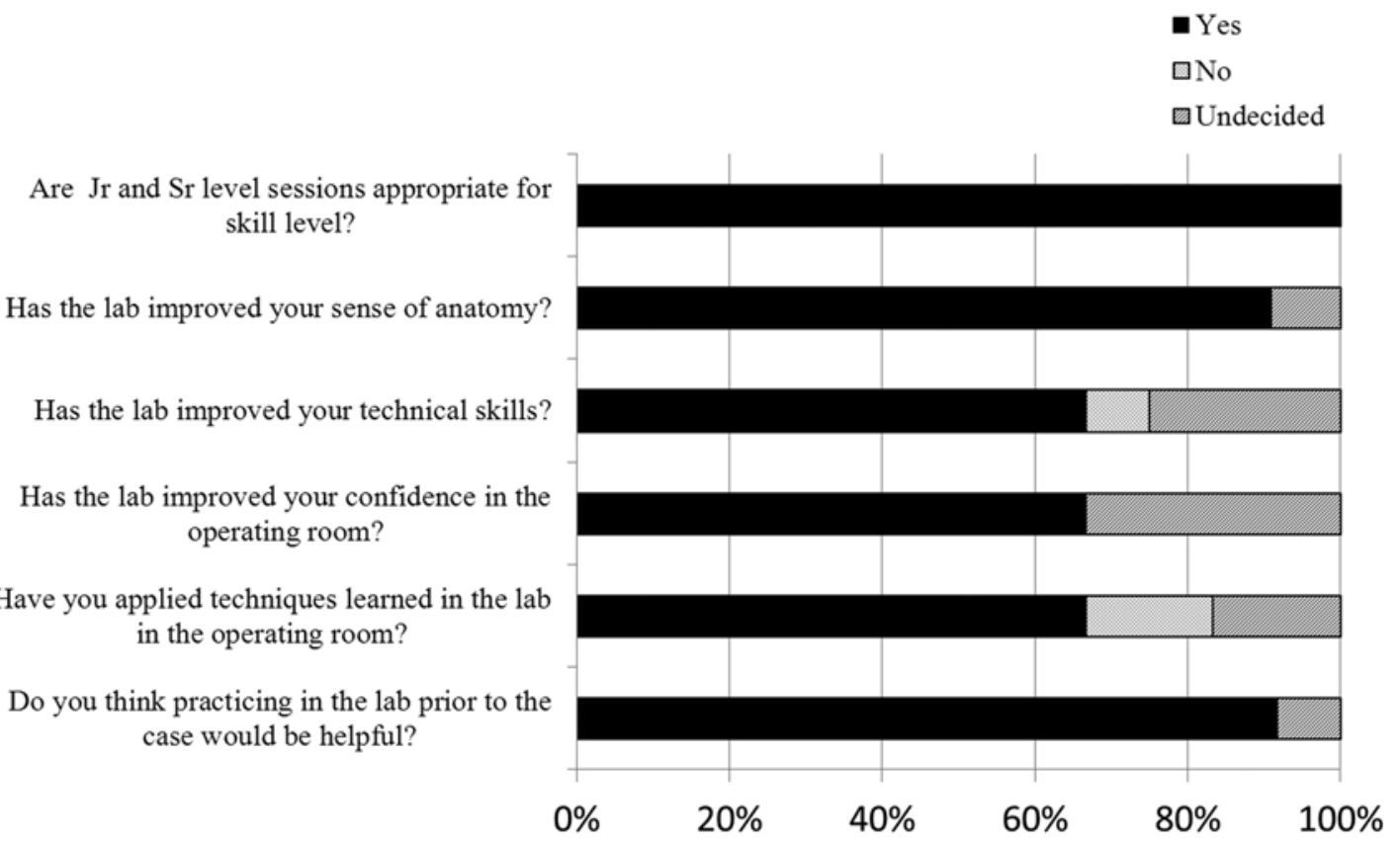

FIG. 2. Results from the resident survey regarding the impact of the surgical skills laboratory and dissection curriculum, which was completed by residents in PGY 2-7 $(n=12)$ who had completed at least 1 year of the curriculum. 
Have duty hours restricted the development of your surgical skills?

If yes, do you think the skills lab can replace the loss in education from duty hours? $\mathrm{N}=4$

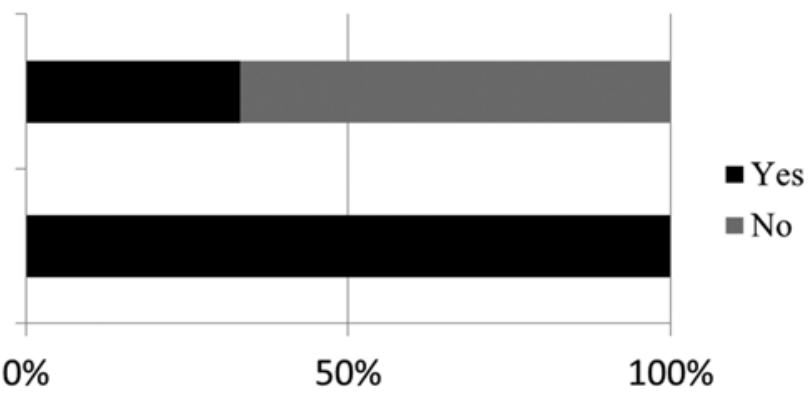

FIG. 3. Resident survey results. Residents were asked about the effects of duty-hour regulations on their training and the potential impact of the surgical skills laboratory.

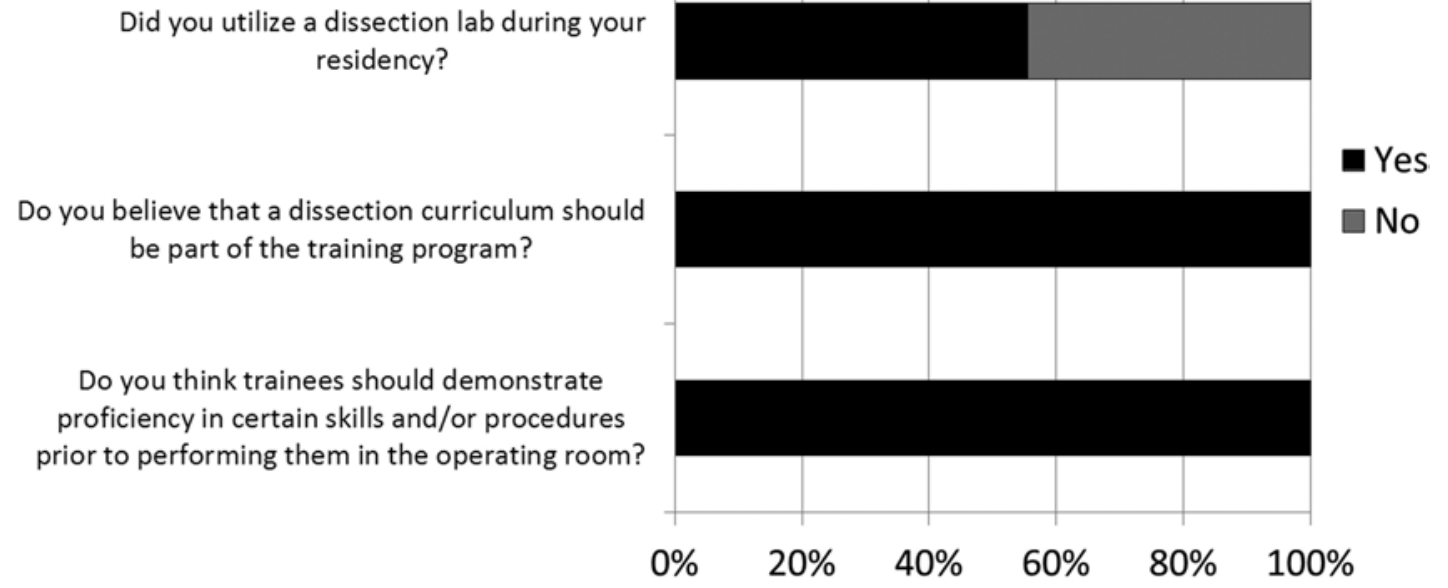

FIG. 4. Faculty survey results. Attending neurosurgeons were surveyed regarding their experience with anatomical dissection during their training and its role in current resident training.

take a step toward creating minimal competencies that each trainee will be mandated to meet.

Finally, the resources of a surgical skills laboratory may become a necessary tool in the future assessment of neurosurgical trainees. In recent years, graduate medical education has trended toward more structured assessment of training competency. The ACGME has established core competency requirements that must be met for all training programs, breaking down the training experience into components that must be fulfilled. This has resulted in the need for a structured environment to refine and assess surgical skills. Some surgical training programs have already established a skills competency program to assess surgical education by having trainees achieve graduated milestones. ${ }^{6}$ In neurosurgery, the recent advent of milestones has forced training programs to be able to document a progression of learning to increase uniformity in training standards. These milestones will likely cross over into the assessment of surgical skills-which is far removed from the apprenticeship-based model in which graduation is a subjective assessment-to a model that focuses on meeting graduated competencies. ${ }^{5}$ Training programs in the future may be required to document the development and competency of the necessary skill sets to graduate as an adequately trained surgeon. A surgical skills laboratory can provide the environment for a systematic simulated component of surgical training and also provide a means of testing the proficiency of the skills acquired.

\section{Conclusions}

The obstacles to creating a surgical skills laboratory at any given institution may appear daunting but can be overcome at any academic institution, regardless of the initial resources available. Nearly 3 years after implementation of the dissection curriculum, we have found a positive response from the trainees. The ultimate gauge of effectiveness for a set curriculum will be from the attending surgeons' assessment of whether trainees are better prepared for operative procedures and more completely trained at the conclusion of their training. This assessment is difficult to do given that it is subjective, which leads to the need for more uniform assessment of surgical training in the future.

\section{References}

1. Aboud E, Al-Mefty O, Yaşargil MG: New laboratory model for neurosurgical training that simulates live surgery. J Neurosurg 97:1367-1372, 2002

2. Alvernia JE, Pradilla G, Mertens P, Lanzino G, Tamargo RJ: Latex injection of cadaver heads: technical note. Neurosurgery 67 (2 Suppl Operative):362-367, 2010

3. Bridges M, Diamond DL: The financial impact of teaching 
surgical residents in the operating room. Am J Surg 177:2832, 1999

4. Cohen-Gadol AA, Piepgras DG, Krishnamurthy S, Fessler RD: Resident duty hours reform: results of a national survey of the program directors and residents in neurosurgery training programs. Neurosurgery 56:398-403, 2005

5. Dutta S, Krummel TM: Simulation: a new frontier in surgical education. Adv Surg 40:249-263, 2006

6. Hammond L, Ketchum J, Schwartz BF: Accreditation council on graduate medical education technical skills competency compliance: urologic surgical skills. J Am Coll Surg 201:454-457, 2005

7. Harvey AM: The influence of William Stewart Halsted's concepts of surgical training. Johns Hopkins Med J 148:215236, 1981

8. Jagannathan J, Vates GE, Pouratian N, Sheehan JP, Patrie J, Grady MS, et al: Impact of the Accreditation Council for Graduate Medical Education work-hour regulations on neurosurgical resident education and productivity. J Neurosurg 110:820-827, 2009

9. Klein I, Steger U, Timmermann W, Thiede A, Gassel HJ: Microsurgical training course for clinicians and scientists at a German University hospital: a 10-year experience. Microsurgery 23:461-465, 2003

10. Krishnamurthy S, Powers SK: The use of fabric softener in neurosurgical prosections. Neurosurgery 36:420-424, 1995

11. Kshettry VR, Mullin JP, Schlenk R, Recinos PF, Benzel EC: The role of laboratory dissection training in neurosurgical residency: results of a national survey. World Neurosurg 82:554-559, 2014

12. Pichierri A, Frati A, Santoro A, Lenzi J, Delfini R, Pannarale
L, et al: How to set up a microsurgical laboratory on small animal models: organization, techniques, and impact on residency training. Neurosurg Rev 32:101-110, 2009

13. Salma A, Chow A, Ammirati M: Setting up a microneurosurgical skull base lab: technical and operational considerations. Neurosurg Rev 34:317-326, 2011

14. Sanan A, Abdel Aziz KM, Janjua RM, van Loveren HR, Keller JT: Colored silicone injection for use in neurosurgical dissections: anatomic technical note. Neurosurgery 45:1267-1274, 1999

15. Shimizu S, Tanaka R, Rhoton AL Jr, Fukushima Y, Osawa S, Kawashima M, et al: Anatomic dissection and classic threedimensional documentation: a unit of education for neurosurgical anatomy revisited. Neurosurgery 58:E1000, 2006

16. Yaşargil MG: A legacy of microneurosurgery: memoirs, lessons, and axioms. Neurosurgery 45:1025-1092, 1999

\section{Author Contributions}

Conception and design: Liu, Kshettry, Recinos. Acquisition of data: Liu, Kshettry. Analysis and interpretation of data: Liu, Kshettry. Drafting the article: Liu, Kshettry. Critically revising the article: Liu, Kshettry, Benzel. Reviewed submitted version of manuscript: Liu, Kshettry, Benzel. Approved the final version of the manuscript on behalf of all authors: Liu. Administrative/ technical/material support: Liu, Kshettry. Study supervision: all authors.

\section{Correspondence}

James K. C. Liu, MetroHealth Medical Center, 2500 MetroHealth Dr., H910, Cleveland, OH 44109. email: jamesliu@ case.edu. 